\title{
Endoscopic treatment including a single-operator peroral cholangioscopy-guided electrohydraulic lithotripsy and endoscopic nasogallbladder drainage combined laparoscopic cholecystectomy for Mirizzi syndrome
}

\author{
Chan PARK ${ }^{1}$, Hyung Ku CHON ${ }^{2}$, Dong Eun PARK ${ }^{* 1}$ \\ 'Department of Hepatobiliary Surgery, Wonkwang University Hospital, Iksan, Korea \\ ${ }^{2}$ Division of Bilopancreas, Department of Internal Medicine, Wonkwang University Hospital, Iksan, Korea
}

Introduction: Mirizzi Syndrome (MS) is rare among gallbladder diseases and may be difficult to treat. In particular, endoscopic treatment often fails owing to unable to access or capture the impacted cystic duct stone. We report a case of MS grade III that were successfully managed by an endoscopic treatment and interval laparoscopic cholecystectomy.

Methods: A 59-year-old women was admitted our hospital presenting with upper abdominal pain and fever up to $38.5^{\circ} \mathrm{C}$. She was mildly jaundiced with scleral icterus with a positive Murphy's sign. An abdomen computed tomography scan and T2-weight magnetic resonance cholangiopancreatography image demonstrated about $1.4 \mathrm{~cm}$ stone at junction of the cystic duct and mid-common bile duct with cholecysto-choledochal fistula, compatible with MS type III and acute calculus cholecystitis.

Results: A digital single-operator cholangioscopy (DSOC)-guided eletrohydraulic lithotripsy (EHL) was performed. Elective laparoscopic cholecystectomy was performed after the stones were completely removed. The patient was discharged without any complications on the 5th day after surgery.

Conclusions: The treatment of MS is mainly surgical and consists of partial or complete cholecystectomy with or without common bile duct exploration. However, in the era of minimal invasive treatment, laparoscopy treatment for low grade MS is being tried steadily. In our case, through preoperative intervention using DSOC guided EHL, the effect of downgrading MS could be achieved, and this resulted in a safe laparoscopic surgical approach. In conclusion, DSOC guided EHL with ENGBD may allow a minimally invasive approach for management of high grade MS. 